\title{
Transverse vibration of single-layer graphene sheets
}

\author{
R Chowdhury ${ }^{1}$, S Adhikari ${ }^{1}$, F Scarpa $^{2}$ and M I Friswell ${ }^{3}$ \\ ${ }^{1}$ Multidisciplinary Nanotechnology Centre, Swansea University, Singleton Park, Swansea SA2 8PP, UK \\ 2 Advanced Composites Centre for Innovation and Science, University of Bristol, Bristol BS8 1TR, UK \\ ${ }^{3}$ School of Engineering, Swansea University, Singleton Park, Swansea SA2 8PP, UK \\ E-mail: S.Adhikari@swansea.ac.uk
}

Received 30 November 2010, in final form 18 February 2011

Published 28 April 2011

Online at stacks.iop.org/JPhysD/44/205401

\begin{abstract}
We investigate the vibrational properties of zigzag and armchair single-layer graphene sheets (SLGSs) using the molecular mechanics (MM) approach. The natural frequencies of vibration and their associated intrinsic vibration modes are obtained. Vibrational analysis is performed with different chirality and boundary conditions. The simulations are carried out for three types of zigzag and armchair SLGS. The universal force field potential is used for the MM approach. The first four natural frequencies are obtained for increasing lengths. The results indicate that the natural frequencies decrease as the length increases. The results follow similar trends with results of previous studies for SLGS using a continuum structural mechanics approach. These results have shown the applicability of SLGSs as electromechanical resonators.
\end{abstract}

S Online supplementary data available from stacks.iop.org/JPhysD/44/205401/mmedia

(Some figures in this article are in colour only in the electronic version)

\section{Introduction}

In 2003, Gan et al [1] described the in situ exfoliation of a single layer with an intersecting grain boundary in graphite using an STM operated in air. The exfoliation technique has led to the successful production of graphene, opening a new era in the field of nanoelectronics [2-4]. The very high in-plane stiffness of graphene sheets [5] has suggested some possible use of graphite nanosheets for nanosensors and NEMS applications $[6,7]$, due to their extremely high surface-to-volume ratio, as well as large deflection capability under point loading [8-13]. In this paper the out-of-plane or transverse vibration of singlelayer graphene sheets (SLGSs) is considered. The vibration studies could be useful for graphene-based mass and/or gas sensors [6, 14, 15-18].

The vibration of single and multiple layer graphene sheets has been investigated by several authors, using continuum mechanics approaches [19], equivalent lattice structures made by atomistic-continuum models representing the $\mathrm{C}-\mathrm{C}$ bonds [20], and molecular dynamics approaches combined with continuum mechanics for thickness identification [21]. The out-of-plane deformation of SLGS has been considered using the continuum mechanics models [13,22], together with continuum and truss-like structural assemblies [23-30]. In a recent paper a nonlinear mechanical model has been used [31] to take account for large deformations in SLGS. They observed higher resonance frequencies from the nonlinear model compared with the equivalent linear model. A molecular mechanics (MM) approach based on the computation of the Hessian matrix and its eigenvalues has been proposed by some authors to describe the structural dynamics of singlewall carbon nanotubes (SWCNTs) [32], and used to validate a lattice structural mechanics (SM) approach in nanoribbons [33]. In this work, we describe the behaviour of the natural frequencies and modeshapes of SLGSs with various boundary conditions using the MM method, and compare the findings with continuum mechanics based on isotropic properties. Kirschoff-based plate formulations consider the graphene sheets as an isotropic continuum, while edge effects and the finite size of the sheets have been demonstrated to provide in-plane special orthotropic properties [5, 29, 34, 35].

The main novelty of our paper is the MM [36,37], which is a higher fidelity model compared with the previously used models $[20,38]$ where the SM approach is used. In the MM approach, the molecule first finds the lowest energy configuration from its pre-optimized state and then the 


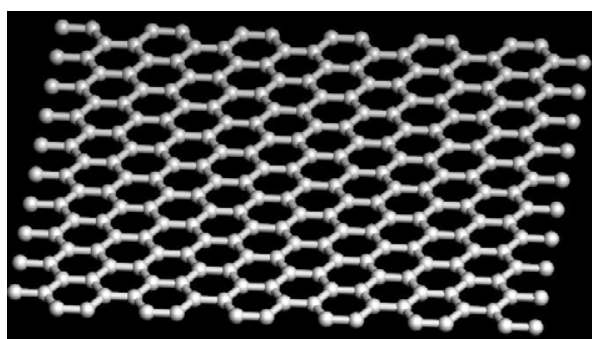

(a) Single layer graphene.

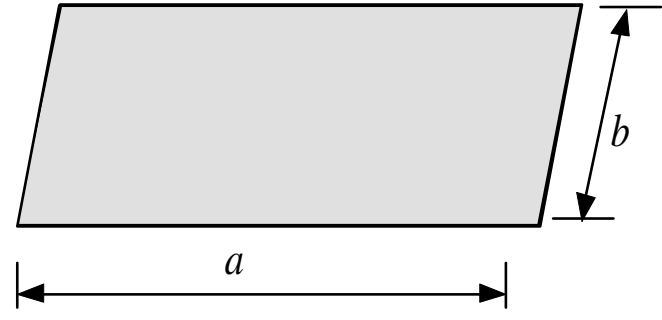

(b) Continuum plate model for graphene.

Figure 1. A rectangular SLGS and its mathematical idealization using a thin continuum plate. The SLGS is assumed to be of dimension $a \times b$.

subsequent calculations are performed. Finding the minimum potential energy surface is a key distinguishing feature in the MM approach. It can be noted that during the optimization the structures may change their configurations. Planar structure such as SLGS may deform to attain the lowest energy configuration. This is ignored in the SM approach [20,38], which only considers the initial geometry of the molecule. In the SM approach the Euler-Bernoulli beam model is used to represent the $\mathrm{C}-\mathrm{C}$ bonds and subsequently the finite element method [39] is used to discretize the equation of motion and obtain the natural frequencies. In the formulation presented in $[20,38]$, the length and the diameter of the 'beams' representing the $\mathrm{C}-\mathrm{C}$ bonds are almost similar (length = $0.142 \mathrm{~nm}$ and diameter $=0.146 \mathrm{~nm}$ ). It is well known [40] that for such 'short beams' the Euler-Bernoulli beam model used in $[20,38]$ produces inaccurate results as the shear deformation is ignored. This can introduce error particularly for the calculation of higher frequencies. The results obtained using the MM approach do not have these drawbacks as neither the Euler-Bernoulli beam model nor the finite element approach is used. In contrast to SM approaches, the MM method also allows the natural frequencies of the system in equilibrium to be computed without any requirement to use an equivalent thickness value for the nanostructure. In general, the introduction of the thickness concept in nanomaterials is highly contentious, leading to the well-known 'Yakobson's paradox' [41,42], responsible for the high scattering of Young's moduli and Poisson's ratio results in the open literature.

We will show that the MM approach is able to capture the equivalent anisotropic properties and their influence in the structural dynamics of the graphene sheets, and therefore provide a valid prediction tool to simulate the resonance behaviour of graphene-based NEMS devices. The paper is organized in the following way. The continuum mechanics approach for the frequency analysis of graphene sheets is presented in section 2 . Section 3 will be centred on the analysis and calculation of the frequencies using a MM model. The numerical results and discussion will be presented in section 4 . Finally, the major conclusions of this paper will be drawn in section 5 based on the results and analyses in section 4 .

\section{Vibration of single-layer graphene}

A SLGS (shown in figure 1) may be approximated by a thin elastic plate [11]. The equation of motion of the transverse free vibration of a thin elastic plate $[40,43]$ can be expressed as

$$
D\left(\frac{\partial^{4} w}{\partial x^{4}}+2 \frac{\partial^{2} w}{\partial x^{2}} \frac{\partial^{2} w}{\partial y^{2}}+\frac{\partial^{4} w}{\partial y^{4}}\right)+\rho \frac{\partial^{2} w}{\partial t^{2}}=0
$$

Here $w \equiv w(x, y, t)$ is the transverse deflection, $x, y$ are coordinates, $t$ is the time, $\rho$ is the mass density per area and the bending rigidity is defined by

$$
D=\frac{E h^{3}}{12\left(1-v^{2}\right)} \text {. }
$$

$E$ is Young's modulus, $h$ is the thickness and $v$ is Poisson's ratio. We consider rectangular graphene sheets with cantilevered (clamped at one edge) and bridged (clamped at two opposite edges) boundary conditions. Following Blevins [44], the natural frequency (in $\mathrm{rad} \mathrm{s}^{-1}$ ) of a rectangular plate of dimension $a \times b$ can be expressed as

$$
\begin{aligned}
\omega_{i j} & =\left\{\frac{\pi^{4} D}{a^{4} \rho}\right\}^{1 / 2}\left\{G_{x}^{4}+G_{y}^{4}\left(\frac{a}{b}\right)^{4}\right. \\
& \left.+2\left(\frac{a}{b}\right)^{2}\left[v H_{x} H_{y}+(1-v) J_{x} J_{y}\right]\right\}^{1 / 2}
\end{aligned}
$$

where $i, j=1,2,3, \ldots$ are mode indices. The values of the coefficients $G_{x}, H_{x}, J_{x}$ and $G_{y}, H_{y}, J_{y}$ depend on the boundary conditions and the mode indices $i, j$. The first set of coefficients depends on the boundary conditions of the edges of width (side $b$ ) while the second set of coefficients depends on the boundary conditions of the edges of length ( $\operatorname{side} a$ ). The boundary conditions on the two edges of length (side $a$ ) are free. In this paper we consider the lower modes of vibration. For the first three modes the coefficients $G_{y}, H_{y}$ and $J_{y}$ are given in table 1 . The coefficients $G_{x}, H_{x}$ and $J_{x}$ for both boundary conditions on the edges of width (side $b$ ) are given in table 2. General expressions of the coefficients for the higher values of $i$ and $j$ are given in [44]. The values given in tables 1 and 2 will be used to obtain the natural frequencies and compare with the MM simulation described in the next section. 
Table 1. Coefficients for the free-free boundary conditions of the edges of length (side $a$ ).

\begin{tabular}{llll}
\hline Mode index $(j)$ & $G_{y}$ & $H_{y}$ & $J_{y}$ \\
\hline 1 & 0 & 0 & 0 \\
2 & 0 & 0 & 1.216 \\
3 & 1.506 & 1.248 & 5.017 \\
\hline
\end{tabular}

Table 2. Coefficients for the two boundary conditions on the edges of width (side $b$ ).

\begin{tabular}{llllllll}
\hline \multirow{2}{*}{$\begin{array}{l}\text { Mode } \\
\text { index }(i)\end{array}$} & \multicolumn{3}{c}{ Clamped-clamped } & & \multicolumn{3}{c}{ Clamped-free } \\
\cline { 2 - 4 } \cline { 6 - 8 } \cline { 6 - 8 } & $G_{x}$ & $H_{x}$ & $J_{x}$ & & $G_{x}$ & $H_{x}$ & $J_{x}$ \\
\hline 1 & 1.506 & 1.248 & 1.248 & & 0.597 & -0.0870 & 0.471 \\
2 & 2.5 & 4.658 & 4.658 & & 1.494 & 1.347 & 3.284 \\
3 & 3.5 & 10.02 & 10.02 & & 2.5 & 4.658 & 7.842 \\
\hline
\end{tabular}

\section{Molecular simulation approach}

Since atomic configurations can have significant impact on the mechanical properties of SLGSs, zigzag and armchair models are adopted in this study. The zigzag and armchair models of graphene sheets under consideration are the following

- Zigzag sheet clamped at one edge (cantilevered condition)

- Zigzag sheet clamped at two opposite edges (bridged condition)

- Armchair sheet clamped at one edge (cantilevered condition)

- Armchair sheet clamped at two opposite edges (bridged condition).

Different atomic configurations and boundary conditions can be considered in an unified manner within the scope of MM. The general expression of total energy is a sum of energies due to valence or bonded interactions and non-bonded interactions [45]

$$
\begin{aligned}
E= & \sum_{0}^{N_{\mathrm{B}}} \frac{1}{2} k_{I J}\left(r-r_{I J}\right)^{2}+\sum_{0}^{N_{\mathrm{A}}} k_{I J K}\left(C_{0}+C_{1} \cos \theta\right. \\
& \left.+C_{2} \cos 2 \theta\right)+\sum_{0}^{N_{\mathrm{T}}} \frac{1}{2} V_{\phi}\left(1-\cos \left(n \phi_{0}\right) \cos (n \phi)\right) \\
& +\sum_{0}^{N_{I}} V_{\omega}\left(C_{0}^{I}+C_{1}^{I} \cos \omega+C_{2}^{I} \cos 2 \omega\right) \\
& +\sum_{0}^{N_{\mathrm{nb}}} R_{I J}\left[-2\left(\frac{x_{I J}}{x}\right)^{6}+\left(\frac{x_{I J}}{x}\right)^{12}\right]+\sum_{0}^{N_{n b}} \frac{q_{I} \cdot q_{J}}{\varepsilon \cdot x} .
\end{aligned}
$$

$N_{\mathrm{B}}, N_{\mathrm{A}}, N_{\mathrm{T}}, N_{\mathrm{I}}$ and $N_{\mathrm{nb}}$ are the numbers of the bond-, angle-, torsion-, inversion- and the non-bonded terms, respectively. $k_{I J}$ and $k_{I J K}$ are the force constants of the bond- and angle terms, respectively. $r$ and $r_{I J}$ are the bond distance and natural bond distance of the two atoms $I$ and $J$, respectively. $\theta$ and $\theta_{0}$ are the angle and natural angle for three atoms $I-J-K$, respectively. $\phi$ and $\phi_{0}$ are the torsion angle and torsion natural angle for three atoms $I-J-K-L$, respectively. $V_{\phi}, n, V_{\omega}, \omega$ are the height of the torsion barrier, periodicity of the torsion potential, height of the inversion barrier and inversion- or outof-plane-angle at atom $I$, respectively. $C_{0}^{I}, C_{1}^{I}$ and $C_{2}^{I}$ are the Fourier coefficients of the inversions terms. $x$ and $x_{I J}$ are the distance and natural distance of two non-bonded atoms $I$ and $J$. $R_{I J}$ is the depth of the Lennard-Jones potential. $q_{I}$ and $\varepsilon$ are the partial charge of atoms $I$ and dielectric constant. For the general nonlinear case, the bend function should have a minimum $\theta=\theta_{0}$, with the second derivative at $\theta_{0}$ equal to the force constant $\left(k_{I J K}\right)$. The Fourier coefficients of the general angle terms $C_{0}, C_{1}$ and $C_{2}$ are evaluated as a function of the natural angle $\theta_{0}$ :

$$
\begin{gathered}
C_{2}=\frac{1}{4 \sin ^{2} \theta_{0}} \\
C_{1}=-4 C_{2} \cos \theta_{0} \\
C_{0}=C_{2}\left(2 \cos ^{2} \theta_{0}+1\right) .
\end{gathered}
$$

The bond stretching force constants $\left(k_{I J}\right)$ are atom based and are obtained from generalization of Badger's rules. The assumption is that the bonding is dominated by attractive ionic terms plus short-range Pauli repulsions [45]. The force constant (in units of $\left(\mathrm{kcal} \mathrm{mol}^{-1}\right) \AA^{-2}$ ) then becomes

$$
k_{I J}=644.12 \frac{Z_{I}^{*} Z_{J}^{*}}{r_{I J}^{3}}
$$

The $Z_{I}^{*}$ is the effective atomic charges, in electron units. Similarly, the angle bend force constants $\left(k_{I J K}\right)$ are generated using the angular generalization of Badger's rule. The force constant (in units of $\mathrm{kcal} \mathrm{mol}^{-1} \mathrm{rad}^{-2}$ ) then becomes [46]

$$
k_{I J K}=644.12 \frac{Z_{I}^{*} Z_{J}^{*}}{r_{I J}^{5}}\left[3 r_{I J} r_{J K}\left(1-\cos ^{2} \theta_{0}\right)-r_{I K}^{2} \cos \theta_{0}\right] \text {. }
$$

The torsional constant $\left(\mathrm{kcal} \mathrm{mol}^{-1}\right)$ is defined as

$$
V_{\phi}=5 \sqrt{U_{I} U_{J}}\left[1+4.18 \ln \left(B O_{J K}\right)\right]
$$

where $B O_{J K}$ is the bond order for atom- $J$ and atom- $K$. $U_{I}$ and $U_{J}$ are the atomic constants defined with UFF $\mathrm{sp}^{2}$. Regarding the inversion term, the coefficients are $C_{0}^{I}=1, C_{1}^{I}=-1$ and $C_{2}^{I}=0$ for $\mathrm{sp}^{2}$ atom type. In this study, we used the UFF model [45], wherein the force field parameters are estimated using general rules based only on the element, its hybridization and its connectivity. Hybridization determines the type of bonding of the carbon atoms with its neighbours. The $\mathrm{sp}^{3}$ hybridization corresponds to the well-known tetrahedral configuration in which carbon binds to four neighbours giving rise to threedimensional inter-connectivity of carbon atoms that is found in diamond. The $\mathrm{sp}^{2}$ bonding in which carbon atoms bind to three neighbours also known as trigonal hybridization gives planar structures found in graphite and graphene. The $\mathrm{sp}^{2}$-hybridized carbon atoms, which are at their energy minimum in planar graphite (or graphene), must be bent to form the closed sphere (fullerenes) or tube (CNT), which produces angle strain. The characteristic reaction of fullerenes is electrophilic addition at 6,6-double bonds, which reduces angle strain by changing $\mathrm{sp}^{2}$-hybridized carbons into $\mathrm{sp}^{3}$-hybridized ones. The change 
in hybridized orbitals causes the bond angles to decrease from about $120^{\circ}$ in the $\mathrm{sp}^{2}$ orbitals to about $109.5^{\circ}$ in the $\mathrm{sp}^{3}$ orbitals. This decrease in bond angles allows for the bonds to bend less when closing the sphere or tube, and thus, the molecule becomes more stable. The force field functional forms and parameters used in this study are in accordance with [45]. The calculation of frequency and their validation for CNTs were detailed in [32]. In the following section we provide the methodology of the frequency calculation.

\subsection{Calculation of the natural frequencies}

We start with the Hessian matrix $f_{\mathrm{CAR}}$, which holds the second partial derivatives of the potential $E$ with respect to the displacement of the atoms in Cartesian coordinates (CAR) [46, 47]:

$$
f_{\mathrm{CAR}_{i j}}=\left(\frac{\partial^{2} E}{\partial \xi_{i} \partial \xi_{j}}\right)_{\mathrm{Opt}} .
$$

This is a $3 N \times 3 N$ matrix ( $N$ is the number of atoms), where $\xi_{1}, \xi_{2}, \xi_{3}, \ldots, \xi_{3 N}$ denote the displacements in Cartesian coordinates, $\Delta x_{1}, \Delta y_{1}, \Delta z_{1}, \ldots, \Delta z_{N}$. The () Opt refers to the fact that the derivatives are taken at the equilibrium positions of the atoms. These force constants are then converted to mass weighted Cartesian coordinates (MWC) [47]

$$
f_{\mathrm{MWC}_{i j}}=\frac{f_{\mathrm{CAR}_{i j}}}{\sqrt{m_{i} m_{j}}}=\left(\frac{\partial^{2} E}{\partial c_{i} \partial c_{j}}\right)_{\mathrm{Opt}}
$$

where $c_{1}=\sqrt{m_{1}} \xi_{1}=\sqrt{m_{1}} \Delta x_{1}, c_{2}=\sqrt{m_{1}} \xi_{2}=\sqrt{m_{1}} \Delta y_{1}$ and so on. $f_{\mathrm{MWC}}$ is diagonalized, yielding a set of $3 N$ eigenvectors and $3 N$ eigenvalues.

The next step is to translate the centre of mass to the origin, and determine the moments and products of inertia, with the goal of finding the matrix that diagonalizes the moment of inertia tensor. Using this matrix we can find the vectors corresponding to the rotations and translations. Once these vectors are known, we know that the rest of the normal modes are vibrations. The centre of mass $\boldsymbol{R}_{\mathrm{COM}}$ is found in the usual way:

$$
\boldsymbol{R}_{\mathrm{COM}}=\frac{\sum_{\alpha} m_{\alpha} \boldsymbol{r}_{\alpha}}{\sum_{\alpha} m_{\alpha}}
$$

where the sums are over the atoms, $\alpha$. The origin is then shifted to the centre of mass $\boldsymbol{r}_{\mathrm{COM}_{\alpha}}=\boldsymbol{r}_{\alpha}-\boldsymbol{R}_{\mathrm{COM}}$. Next we have to calculate the moments of inertia (the diagonal elements) and the products of inertia (off diagonal elements) of the moment of inertia tensor $(\boldsymbol{I})$.

$\boldsymbol{I}=\left(\begin{array}{ccc}I_{x x} & I_{x y} & I_{x z} \\ I_{y x} & I_{y y} & I_{y z} \\ I_{z x} & I_{z y} & I_{z z}\end{array}\right)$

$=\left(\begin{array}{ccc}\sum m_{\alpha}\left(y_{\alpha}^{2}+z_{\alpha}^{2}\right) & -\sum m_{\alpha}\left(x_{\alpha} y_{\alpha}\right) & -\sum m_{\alpha}\left(x_{\alpha} z_{\alpha}\right) \\ -\sum m_{\alpha}\left(y_{\alpha} x_{\alpha}\right) & \sum m_{\alpha}\left(x_{\alpha}^{2}+z_{\alpha}^{2}\right) & -\sum m_{\alpha}\left(y_{\alpha} z_{\alpha}\right) \\ -\sum m_{\alpha}\left(z_{\alpha} x_{\alpha}\right) & -\sum m_{\alpha}\left(z_{\alpha} y_{\alpha}\right) & \sum m_{\alpha}\left(x_{\alpha}^{2}+x_{\alpha}^{2}\right)\end{array}\right)$.

The sums appearing in the above expression is over the index $\alpha$. This symmetric matrix is diagonalized, yielding the principal moments (the eigenvalues $\boldsymbol{I}^{\prime}$ ) and a $3 \times 3$ matrix $(\boldsymbol{X})$, which is made up of the normalized eigenvectors of (I). The eigenvectors of the moment of inertia tensor are used to generate the vectors corresponding to translation and infinitesimal rotation of the molecular system. A Schmidt orthogonalization is used to generate $N_{\text {vib }}=3 N-6$ remaining vectors, which are orthogonal to the six rotational and translational vectors. The result is a transformation matrix $D$ which transforms from mass weighted Cartesian coordinates $\boldsymbol{q}$ to internal coordinates $\boldsymbol{S}=\boldsymbol{D} \boldsymbol{q}$, where rotation and translation have been separated out. Now that we have coordinates in the rotating and translating frame, we need to transform the Hessian, $f_{\mathrm{MWC}}$, to these new internal coordinates (INT) $[46,47]$. Only the $N_{\text {vib }}$ coordinates corresponding to internal coordinates will be diagonalized, although the full $3 N$ coordinates are used to transform the Hessian. The transformation is straightforward as follows:

$$
\boldsymbol{f}_{\mathrm{INT}}=\boldsymbol{D}^{\mathrm{T}} \boldsymbol{f}_{\mathrm{MWC}} \boldsymbol{D} \text {. }
$$

The $N_{\text {vib }} \times N_{\text {vib }}$ submatrix of $\boldsymbol{f}_{\text {INT }}$, which represents the force constants internal coordinates, is diagonalized yielding $N_{\text {vib }}$ eigenvalues $\lambda=4 \pi^{2} \omega^{2}$, and $N_{\text {vib }}$ eigenvectors. If we call the transformation matrix composed of the eigenvectors $L$, then we have

$$
\boldsymbol{L}^{\mathrm{T}} \boldsymbol{f}_{\mathrm{INT}} \boldsymbol{L}=\boldsymbol{\Lambda}
$$

where $\Lambda$ is the diagonal matrix with eigenvalues $\lambda_{i}$. At this point, the eigenvalues need to be converted to frequencies (in $\mathrm{Hz}$ ) as

$$
\omega_{i}=\sqrt{\frac{\lambda_{i}}{4 \pi^{2}}}
$$

\section{Results and discussions}

The resonant frequencies of single-layer graphene (SLG)based resonators depend on the geometric configurations. The atomic structures of SLGS could also exert significant influence on their vibration behaviours. Thus, in this work, we analyse two groups of SLG resonators, i.e. three zigzag SLGS $(10,0),(14,0),(18,0)$ and three armchair SLGS $(11,11)$, $(15,15),(19,19)$, with increasing length. In this study, we computed our results using bridged (atoms at the two sides along width are restrained) and cantilevered (atoms at one side along width are restrained) boundary conditions. The computational results of the first four vibrational frequencies of these zigzag SLGS are calculated and presented in figure 2 and figure 3, respectively, for bridged and cantilevered boundary condition. Similarly, figure 4 and figure 5, respectively, presents the first four vibrational frequencies of these armchair SLGS, for bridged and cantilevered boundary condition. The widths of the SLGS are given in the figure captions.

\subsection{Dependence of the length}

As shown in figures 2 and 3, for SLGS with the length rising from around $20 \AA$ to $120 \AA$, the fundamental frequencies are in the ranges $100-3000 \mathrm{GHz}$ (refer table 3) and $4-1300 \mathrm{GHz}$ (refer table 4) for the zigzag SLGS with bridged and 

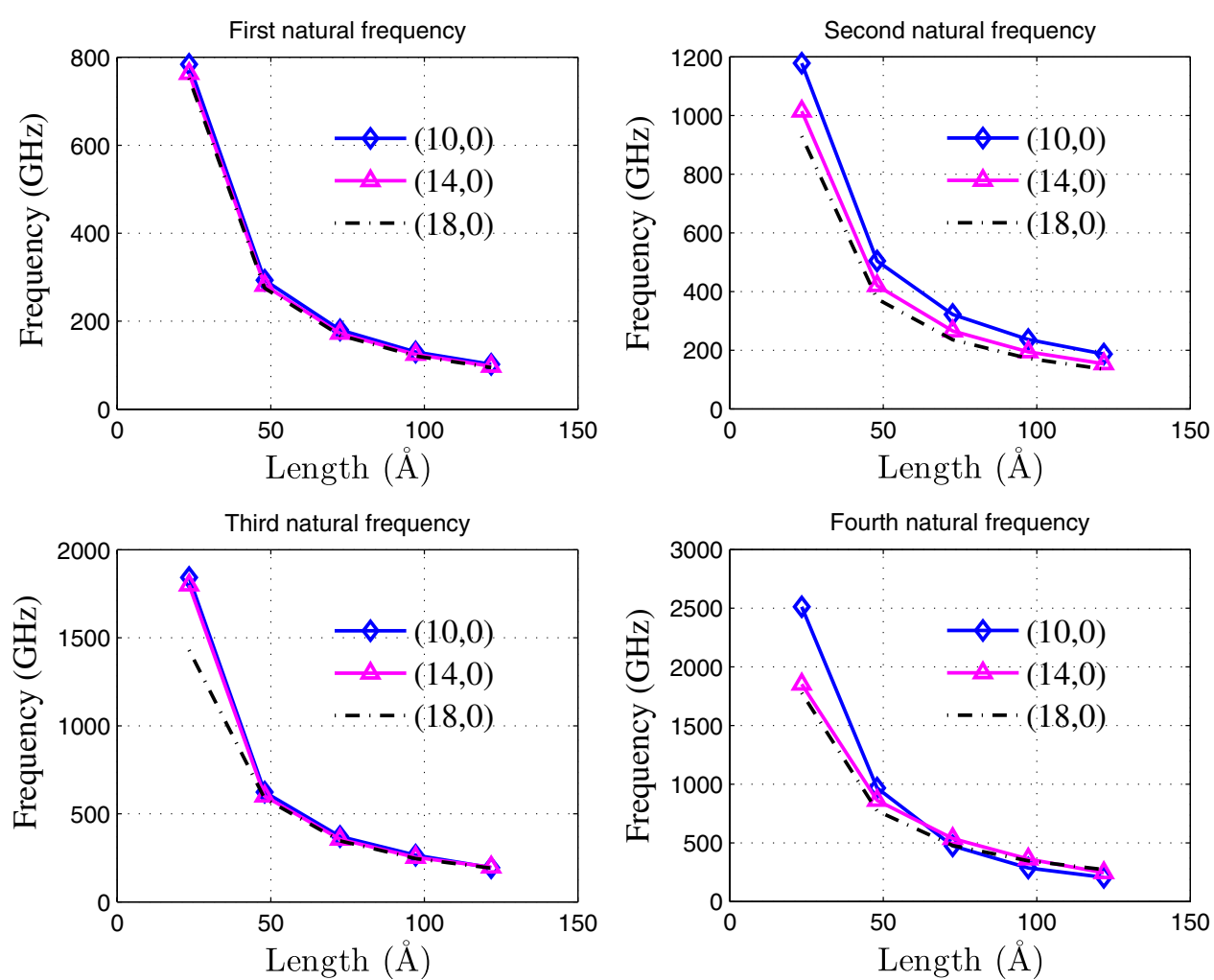

Figure 2. Bridged boundary condition-first four vibrational frequencies of zigzag SLGS as a function of the length of SLGS. The widths are (10,0): $9.317 \AA$; $(14,0): 13.554 \AA$; $(18,0): 17.803 \AA$.
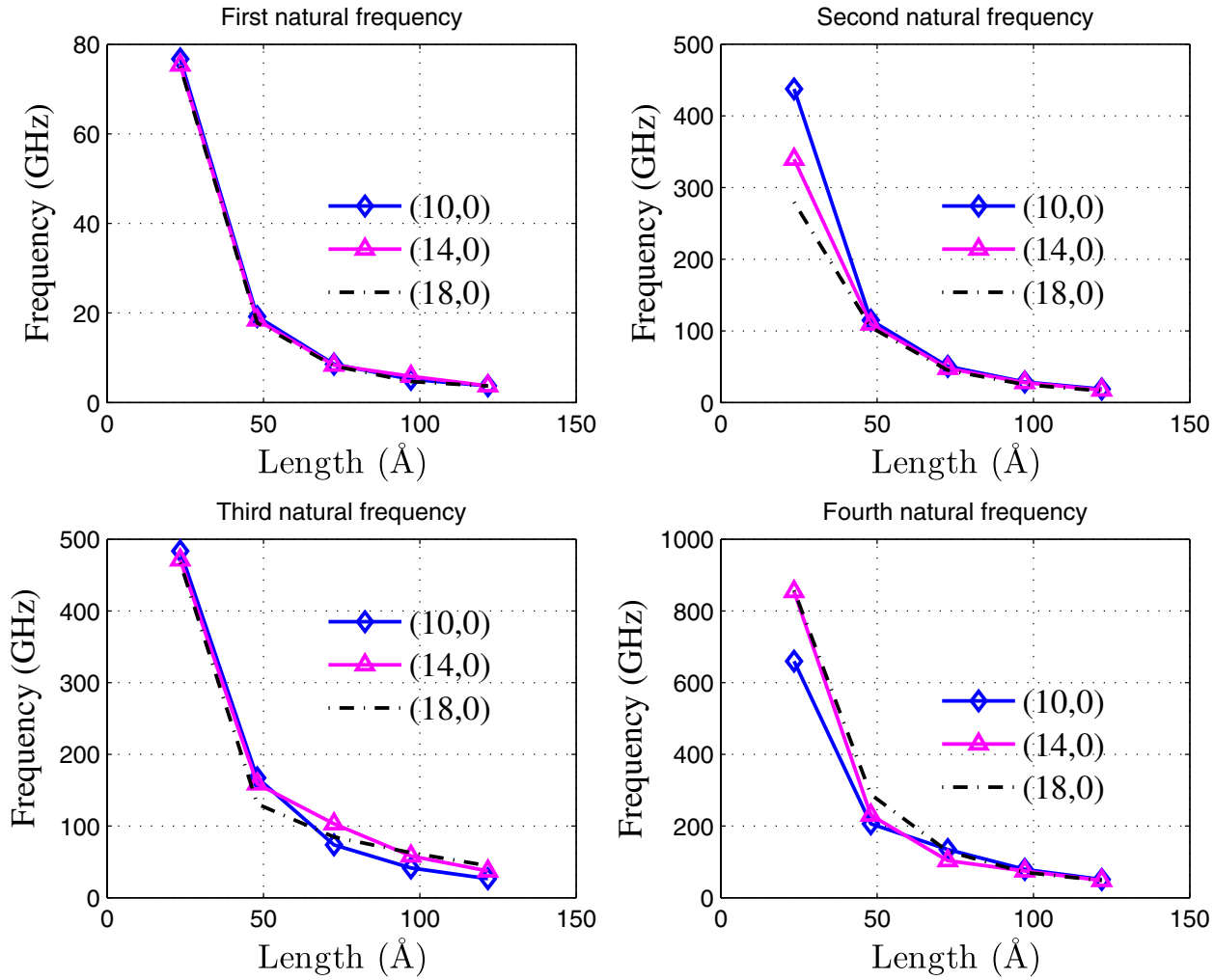

Figure 3. Cantilevered boundary condition-first four vibrational frequencies of zigzag SLGS as a function of the length of SLGS. The widths are (10,0): $9.317 \AA$; $(14,0): 13.554 \AA$; $(18,0)$ : $17.803 \AA$. 

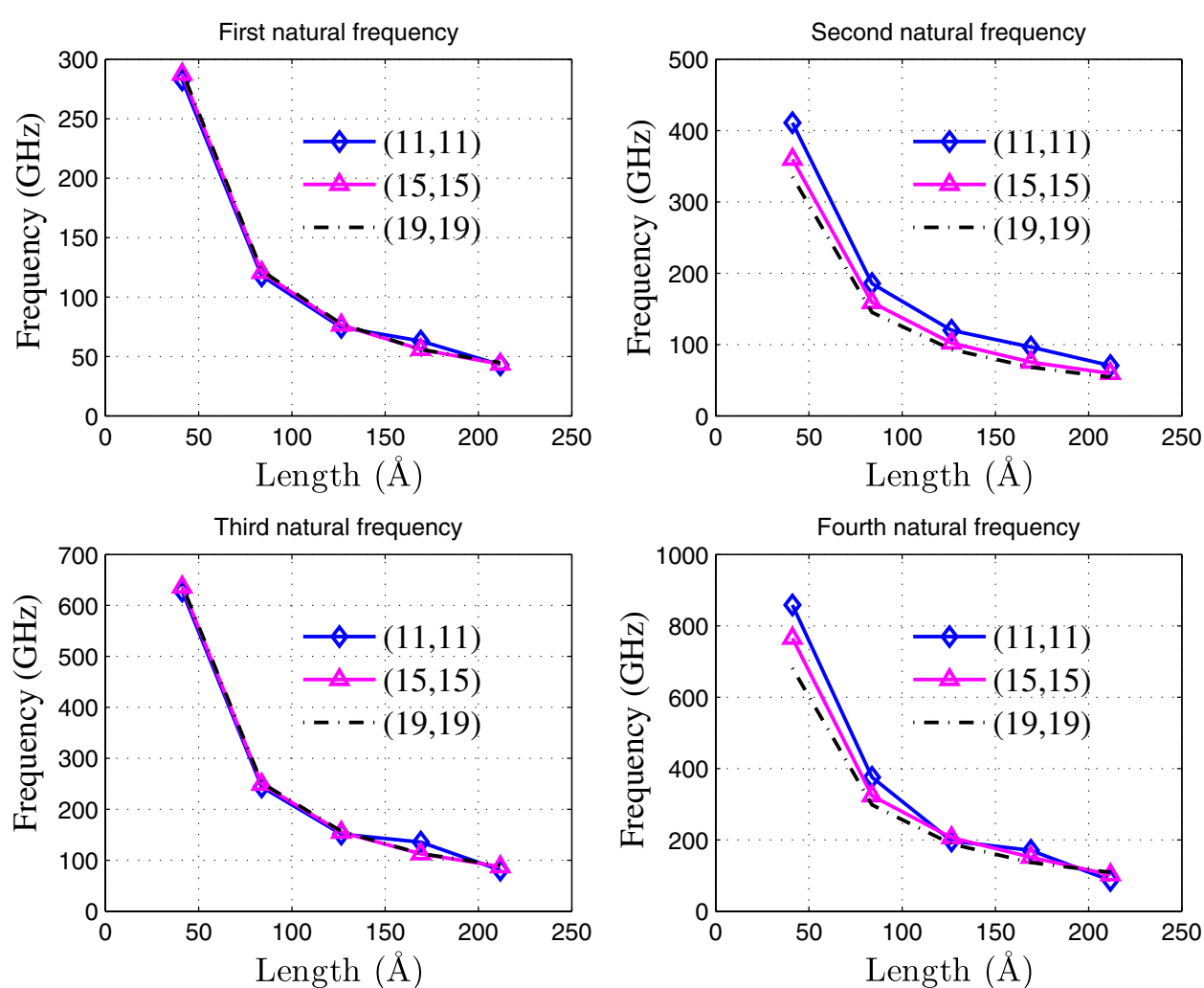

Figure 4. Bridged boundary condition-first four vibrational frequencies of armchair SLGS as a function of the length of SLGS. The widths are (11,11): $12.31 \AA$; $(15,15): 17.23 \AA$; $(19,19): 122.15 \AA$.
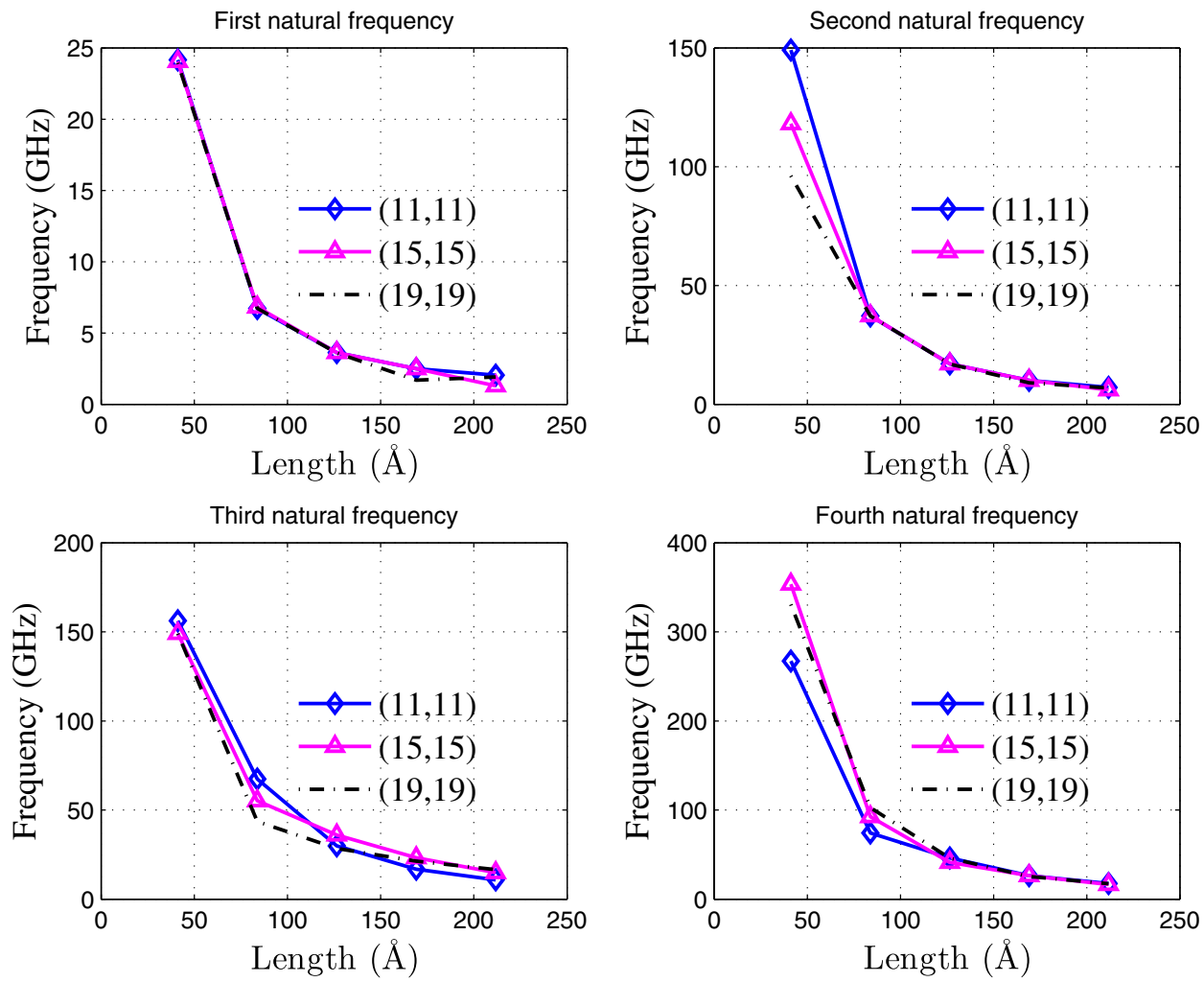

Figure 5. Cantilevered boundary condition - first four vibrational frequencies of armchair SLGS as a function of the length of SLGS. The widths are (11,11): $12.31 \AA$; $(15,15)$ : $17.23 \AA$; $(19,19)$ : $122.15 \AA$. 
Table 3. Vibrational frequencies of zigzag SLGS in GHz—bridged boundary condition.

\begin{tabular}{lccrrrr}
\hline Index and width $(\AA)$ & Length $(\AA)$ & \multicolumn{1}{l}{$\omega_{1}$} & \multicolumn{1}{l}{$\omega_{2}$} & \multicolumn{1}{l}{$\omega_{3}$} & \multicolumn{1}{l}{$\omega_{4}$} & \multicolumn{1}{l}{$\omega_{5}$} \\
\hline & 23.391 & 784.10 & 1178.43 & 1842.16 & 2511.38 & 2960.40 \\
& 47.995 & 293.30 & 504.48 & 623.61 & 967.17 & 1006.04 \\
$(10,0)$ & 72.603 & 180.15 & 321.84 & 370.86 & 474.31 & 580.16 \\
9.317 & 97.213 & 130.12 & 236.47 & 264.56 & 285.31 & 406.86 \\
& 121.822 & 101.88 & 186.91 & 194.26 & 205.92 & 313.98 \\
& 23.391 & 763.00 & 1014.94 & 1799.25 & 1850.99 & 2220.43 \\
& 47.995 & 281.72 & 420.08 & 600.04 & 861.22 & 983.66 \\
$(14,0)$ & 72.603 & 172.47 & 265.86 & 355.04 & 536.70 & 558.29 \\
13.554 & 97.213 & 124.42 & 194.62 & 252.85 & 361.47 & 387.81 \\
& 121.822 & 97.34 & 153.53 & 196.67 & 244.08 & 299.77 \\
& 23.391 & 752.30 & 929.62 & 1430.21 & 1777.36 & 2059.21 \\
$(18,0)$ & 47.995 & 275.09 & 374.56 & 587.23 & 772.02 & 897.41 \\
17.803 & 72.603 & 168.02 & 235.48 & 345.94 & 477.20 & 544.22 \\
& 97.213 & 121.12 & 171.99 & 246.01 & 346.27 & 379.20 \\
& 121.822 & 94.72 & 135.45 & 191.26 & 272.03 & 291.82 \\
\hline
\end{tabular}

Table 4. Vibrational frequencies of zigzag SLGS in GHz—cantilevered boundary condition.

\begin{tabular}{lrrrrrr}
\hline Index and width $(\AA)$ & Length $(\AA)$ & \multicolumn{1}{l}{$\omega_{1}$} & \multicolumn{1}{l}{$\omega_{2}$} & \multicolumn{1}{l}{$\omega_{3}$} & \multicolumn{1}{l}{$\omega_{4}$} & \multicolumn{1}{l}{$\omega_{5}$} \\
\hline & 23.391 & 76.71 & 437.63 & 483.34 & 659.82 & 1323.45 \\
& 47.995 & 19.18 & 115.03 & 166.98 & 206.05 & 321.91 \\
$(10,0)$ & 72.603 & 8.58 & 50.46 & 73.69 & 134.18 & 140.80 \\
9.317 & 97.213 & 5.20 & 28.57 & 41.25 & 78.61 & 99.77 \\
& 121.822 & 3.75 & 18.70 & 26.34 & 50.53 & 79.32 \\
& 23.391 & 75.35 & 339.71 & 471.07 & 854.05 & 1035.23 \\
& 47.995 & 18.41 & 109.56 & 158.49 & 230.04 & 311.10 \\
$(14,0)$ & 72.603 & 8.34 & 47.75 & 102.90 & 103.10 & 133.99 \\
13.554 & 97.213 & 5.84 & 27.91 & 58.08 & 74.40 & 77.05 \\
& 121.822 & 3.74 & 17.52 & 37.11 & 47.57 & 60.62 \\
& 23.391 & 75.00 & 279.65 & 467.10 & 857.12 & 883.47 \\
& 47.995 & 17.80 & 104.88 & 130.68 & 287.79 & 305.18 \\
$(18,0)$ & 72.603 & 8.15 & 45.04 & 84.69 & 128.84 & 131.38 \\
17.803 & 97.213 & 4.68 & 24.89 & 62.71 & 70.16 & 74.43 \\
& 121.822 & 3.67 & 16.34 & 44.52 & 47.76 & 49.86 \\
\hline
\end{tabular}

cantilevered boundary conditions, respectively. While for the armchair SLGS, the variation of frequencies is between 40 and $1070 \mathrm{GHz}$ (refer table 5) and 2 and $415 \mathrm{GHz}$ (refer table 6) for bridged and cantilevered boundary conditions, respectively, with the length rising from around $40 \AA$ to $210 \AA$. The trends of the frequency changes with length are generally in accordance with that given in the literature [20]. The discrepancy is primarily a result of the different end constraints, geometric configurations of SLGS and the differences in the simulation approaches. Recall that here the MM approach is used as opposed to the finite element method employed in [20,38].

\subsection{Dependence of the geometric configuration}

For both zigzag and armchair SLGS, the frequencies of all five modes generally decrease with increasing length. The curves of frequency becomes steeper for the SLGS of smaller sheet length $(\leqslant 50 \AA)$. This shows that the dependence on the aspect ratio is stronger for the frequencies of shorter SLGS. In the meantime, it is also seen from figures 2 and 4 that, for a given length the frequencies of SLGS always decline with rising sheet width. This issue can be further clarified from tables 3 to 6. The frequencies of small-width SLGS are always higher than the corresponding frequencies of large-width SLGS. This is especially so when the length is relatively small. However, the effect of width on the frequencies diminishes for SLGS with larger widths. As an example, for zigzag SLGS (refer table 3) with width $9.317 \AA$ the frequency decreases from 784.10 to $101.88 \mathrm{GHz}$ when the length increases from 23.391 to $121.822 \AA$, while for certain length of $23.391 \AA$ it only varies from 784.10 to $752.30 \mathrm{GHz}$, when width increases from 9.317 to $17.803 \AA$. Similar behaviour is also observed for armchair SLGS in table 3. Thus we see that when the aspect ratio of the SLGS grows, the difference in frequency due to the variation of length decreases significantly whereas the ratio between the frequencies remains almost unchanged. This observation suggests that the influence of width on the vibration frequency of SLGS does not significantly change with increasing length. Here the decreasing frequencies with increasing length and width observed in figure 2, and figure 4 can be attributed to the fact that SLGS of larger length and width possess lower dynamic structural stiffness in both longitudinal and transverse directions. In particular, for SLGS of small width their transverse stiffness is high. The frequency of such SLGS thus becomes more sensitive to their longitudinal rigidity, which finally leads to stronger effect of the length for SLGS with smaller width. 
Table 5. Vibrational frequencies of armchair SLGS in GHz-bridged boundary condition.

\begin{tabular}{lrrrrrr}
\hline Index and width $(\AA)$ & Length $(\AA)$ & \multicolumn{1}{l}{$\omega_{1}$} & \multicolumn{1}{l}{$\omega_{2}$} & \multicolumn{1}{l}{$\omega_{3}$} & \multicolumn{1}{l}{$\omega_{4}$} & \multicolumn{1}{l}{$\omega_{5}$} \\
\hline & 41.21 & 282.84 & 411.22 & 628.37 & 858.54 & 1072.30 \\
$(11,11)$ & 83.83 & 117.86 & 185.65 & 243.91 & 375.70 & 385.14 \\
12.31 & 126.46 & 74.47 & 120.09 & 151.31 & 197.16 & 232.77 \\
& 169.08 & 63.05 & 96.48 & 135.33 & 170.34 & 176.12 \\
& 211.71 & 42.94 & 70.46 & 80.55 & 86.31 & 130.72 \\
& 41.21 & 287.54 & 359.84 & 636.08 & 765.41 & 953.01 \\
$(15,15)$ & 83.83 & 120.58 & 158.87 & 248.98 & 323.45 & 392.11 \\
17.23 & 126.46 & 76.32 & 102.08 & 154.90 & 205.84 & 237.93 \\
& 169.08 & 55.85 & 75.30 & 112.58 & 151.24 & 151.67 \\
& 211.71 & 43.43 & 59.43 & 87.37 & 101.74 & 119.02 \\
$(19,19)$ & 41.21 & 290.01 & 335.32 & 640.24 & 681.45 & 720.46 \\
22.15 & 83.83 & 122.14 & 145.19 & 251.80 & 297.89 & 395.86 \\
& 126.46 & 77.40 & 93.09 & 156.92 & 188.19 & 240.78 \\
& 169.08 & 55.91 & 68.18 & 112.83 & 136.68 & 171.43 \\
& 211.71 & 44.70 & 54.26 & 89.80 & 108.84 & 123.21 \\
\hline
\end{tabular}

Table 6. Vibrational frequencies of armchair SLGS in GHz-cantilevered boundary condition.

\begin{tabular}{lrrrrrr}
\hline Index and width $(\AA)$ & Length $(\AA)$ & \multicolumn{1}{c}{$\omega_{1}$} & \multicolumn{1}{c}{$\omega_{2}$} & \multicolumn{1}{c}{$\omega_{3}$} & \multicolumn{1}{c}{$\omega_{4}$} & \multicolumn{1}{c}{$\omega_{5}$} \\
\hline & 41.21 & 24.18 & 149.23 & 156.26 & 267.23 & 416.07 \\
$(11,11)$ & 83.83 & 6.72 & 37.14 & 67.35 & 74.23 & 102.89 \\
12.31 & 126.46 & 3.63 & 17.02 & 29.88 & 46.14 & 48.69 \\
& 169.08 & 2.50 & 10.08 & 16.82 & 26.42 & 36.25 \\
& 211.71 & 2.04 & 7.06 & 10.81 & 17.54 & 28.85 \\
& 41.21 & 24.06 & 118.14 & 149.10 & 353.65 & 387.94 \\
$(15,15)$ & 83.83 & 6.82 & 37.24 & 55.23 & 92.43 & 102.87 \\
17.23 & 126.46 & 3.62 & 16.98 & 36.00 & 41.29 & 46.08 \\
& 169.08 & 2.50 & 10.02 & 23.26 & 26.35 & 26.76 \\
& 211.71 & 1.29 & 6.03 & 14.86 & 16.47 & 21.08 \\
$(19,19)$ & 41.21 & 23.96 & 96.16 & 148.95 & 330.70 & 413.55 \\
22.15 & 83.83 & 6.73 & 37.20 & 43.58 & 102.76 & 116.43 \\
& 126.46 & 3.59 & 16.90 & 28.47 & 45.93 & 52.51 \\
& 169.08 & 1.91 & 9.04 & 21.27 & 25.46 & 29.60 \\
& 211.71 & 1.69 & 6.82 & 16.53 & 17.27 & 19.03 \\
\hline
\end{tabular}

Based on the MM method, mode shapes of the SLGS are obtained. The first six mode shapes of zigzag and armchair sheets are given in the supplementary document (see supplementary materials stacks.iop.org/JPhysD/44/165408/mmedia). The first mode shape plays a significant role in the design of the nanomechanical resonators. It is perceived that the SLGS with different boundary conditions has a sinusoidal and/or hyperbolic sine and cosine configuration. These configurations guarantee the ease of detection of any small deflection in the SLGS. In addition, mode shapes of the SLGS, in contrast to the CNTs, are not changed by the length or aspect ratio.

\subsection{Dependence of the atomic structure}

Next we examine the effect of atomic structures on the frequency of SLGS. To this end we consider the calculated frequencies in table 5 and table 3 respectively, for armchair and zigzag SLGS with the bridged boundary condition. Similarly, we consider the calculated frequencies in table 6 , and table 4 respectively, for armchair and zigzag SLGS with the cantilevered boundary condition. The results in figure 6 show that the chirality does not have a significant influence
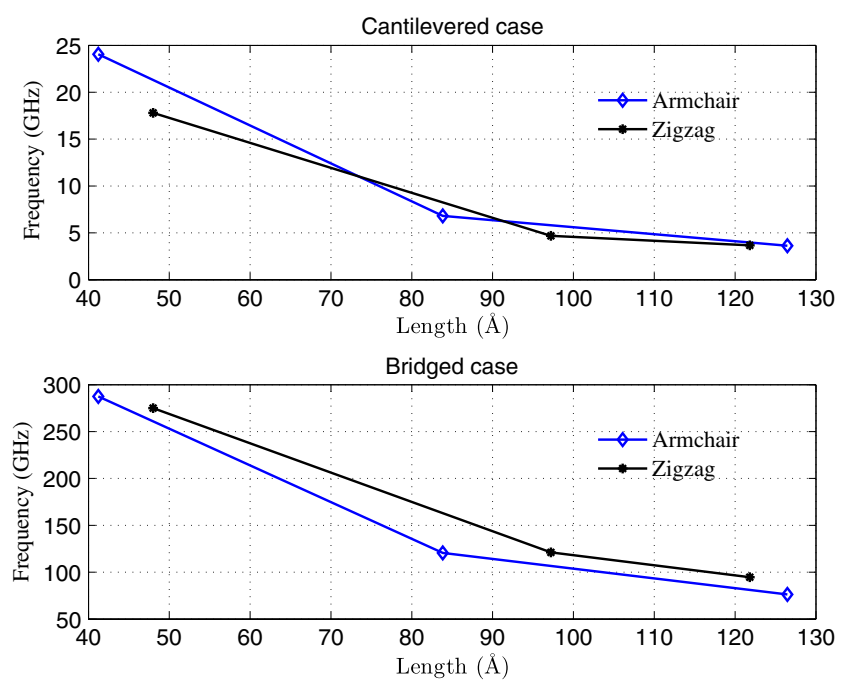

Figure 6. Dependence of the molecular structure on the fundamental frequency of SLGS. It is found that natural frequencies of zigzag SLGS $(18,0)$ are higher compared with armchair SLGS $(15,15)$ for bridged case, whereas for cantilevered case, it is almost comparable. 


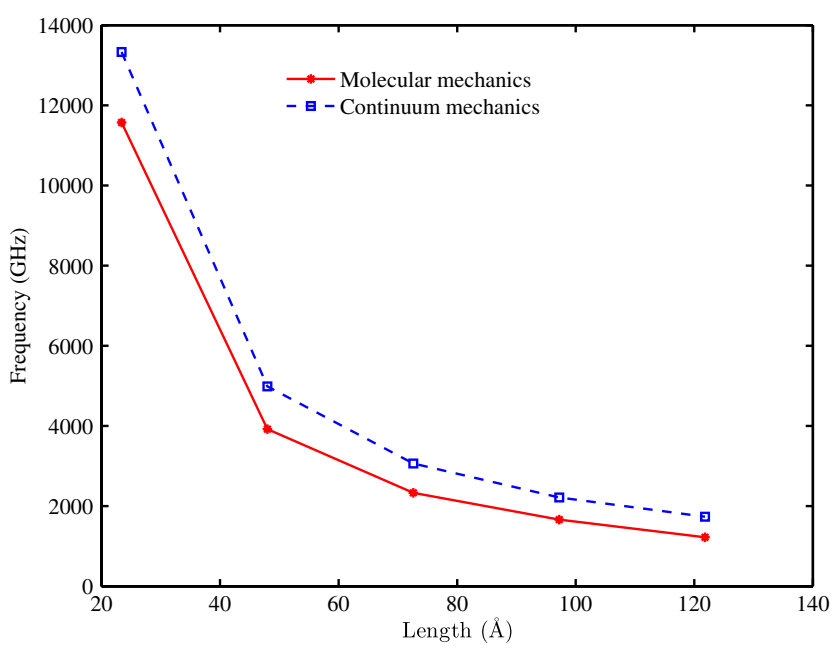

(a) Frequency of the second bending mode.

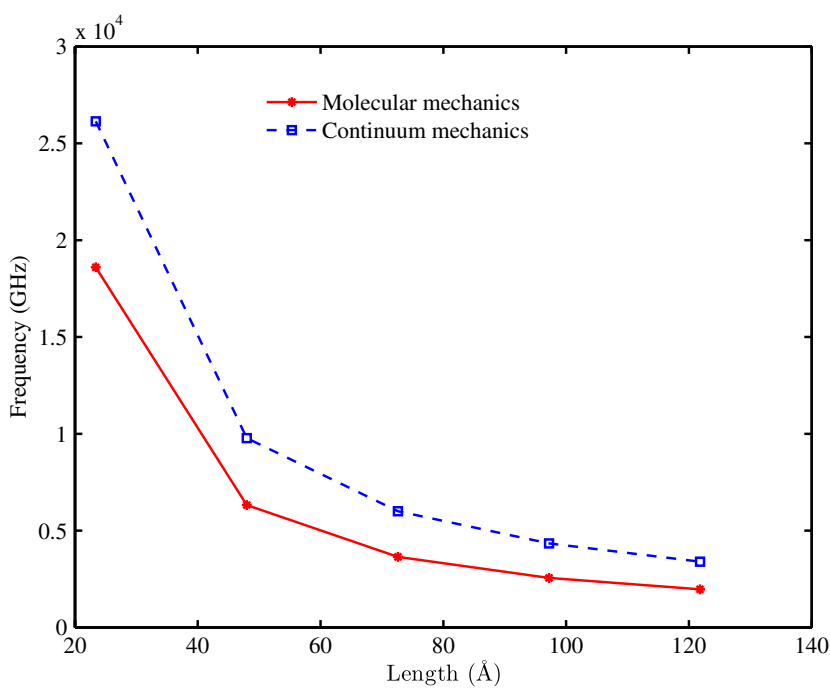

(b) Frequency of the third bending mode.

Figure 7. Bridged boundary condition-vibrational frequencies of the zigzag SLGS $(10,0)$ for the second and third bending mode. MM results and continuum mechanics results are compared for different values of the length.

on the natural frequencies of vibration. It is shown that for almost the same width and length the fundamental frequencies of zigzag SLGS are only higher than those of armchair SLGS for the bridged case, whereas for the cantilevered case, it is almost comparable. In the context of SWCNTs, the difference between the frequencies of the two types (e.g. zigzag and armchair) is also not very large [32]. This may be expected as SWCNTs are effectively rolled up SLGS. The frequency of SLGS is primarily determined by their geometry, i.e. length and the aspect ratio, and cannot be substantially changed by varying their atomic structure. This finding demonstrates that the continuum models can produce a good approximation for the vibration of SLGS with different atomic structures.

\subsection{Comparison with the continuum theory}

In this section we investigate whether the vibrational frequencies obtained from the simple continuum plate model are comparable to the vibrational frequencies computed using the MM approach. The density per unit area on the SLGS is computed from the total mass divided by the total area on the SLGS. We use the first natural frequency to obtain the bending rigidity $D$ using the natural frequency equation (3) with the values of the coefficients corresponding to $i=1$ and $j=1$ in tables 1 and 2 . We then use this value of $D$ to compute the higher natural frequencies to understand if the simple plate model is applicable. The first natural frequency is not shown in the figures because the constant $D$ is calculated using the first natural frequency.

In figure 7, the second and third bending mode frequencies are compared for the bridged boundary condition. We used $(10,0)$ zigzag SLGS as an example. The results for the other types of SLGS used in this study show similar behaviour. The analytical results shown in figure 7 are obtained from equation (3) with the values of the coefficients corresponding to $i=2,3$ for the clamped-clamped case in table 2 and $j=1$ in table 1 . The trend in the variation of the frequencies with respect to the length is similar for both the methods. The difference between the two theories is more prominent for SLGS with smaller dimension. This is expected as the continuum theory may not be very suitable for SLGS in this case. The continuum model tends to overestimate the MM predictions (17\% and $37 \%$ for the second and third mode, respectively). The continuum mechanics formulation assumes an isotropic equivalent material for the graphene. However, edge effects have been demonstrated to play a significant role in the static $[29,34,35,48]$ and dynamic [33] mechanical properties of SLGS, leading to an equivalent orthotropic, rather than isotropic material model for the graphene. Although the dimensions of the SLGS considered in this work are dissimilar, we note a general agreement in terms of magnitude between the eigenvalues calculated with our MM approach, and the results using the MM3 potential in [21].

Figure 8 shows equivalent plots for the SLGS with cantilevered boundary condition. The analytical results shown in figure 8 are obtained from equation (3) with the values of the coefficients corresponding to $i=2,3$ for the clampedfree case in table 2 and $j=1$ in table 1 . The results predicted by the two approaches agree more closely for this boundary condition. The results obtained here shows that the boundary condition has an effect on the accuracy of the predictions from the continuum theory.

\section{Conclusions}

The vibrational properties of zigzag and armchair single-wall graphene sheets (SLGS) are studied. A molecular mechanics based approach is used to estimate the frequencies. We used the UFF model, wherein the force field parameters are estimated using general rules based only on the element, its hybridization, and its connectivity. Two types of boundary conditions are considered, namely, cantilevered and bridged. First five natural frequencies are calculated for three zigzag, namely $(10,0),(14,0)$ and $(18,0)$ and three armchair, namely 


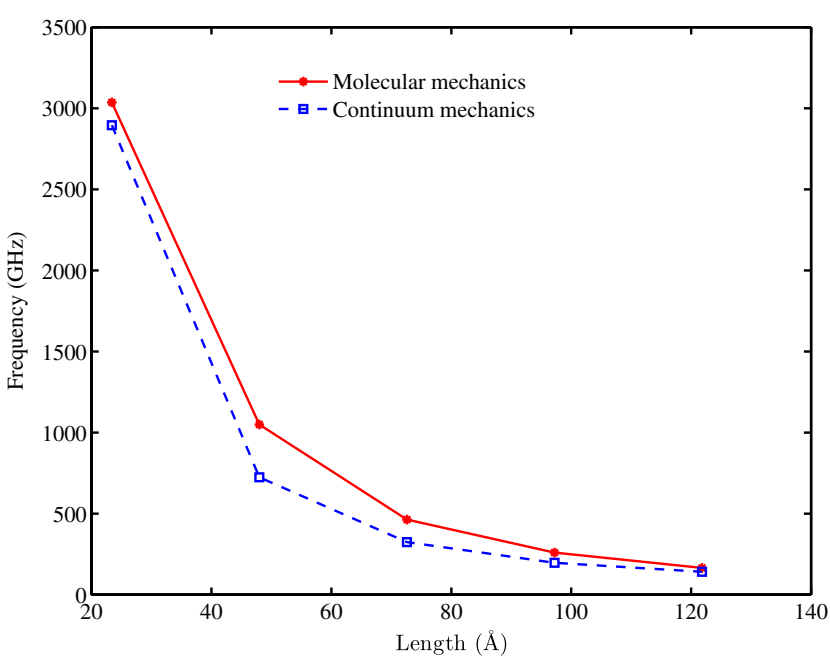

(a) Frequency of the second bending mode.

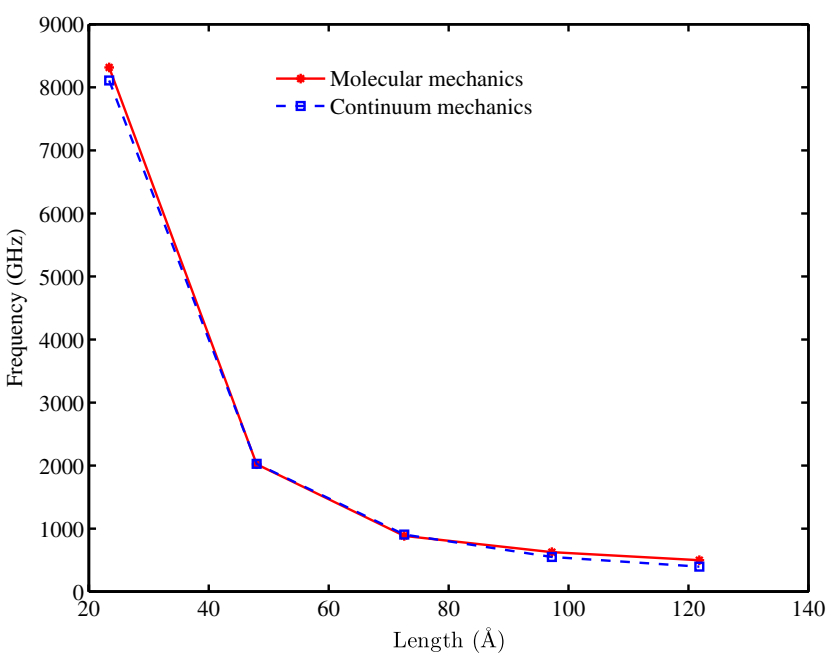

(b) Frequency of the third bending mode.

Figure 8. Cantilevered boundary condition-vibrational frequencies of the zigzag SLGS $(10,0)$ for the second and third bending mode. $\mathrm{MM}$ results and continuum mechanics results are compared for different values of the length.

$(11,11),(15,15)$ and $(19,19)$ SLGS. The natural frequencies of SLGS decrease with length but they are generally insensitive to the atomic structure. Results obtained from the molecular mechanics approach are compared with the same obtained using the continuum plate theory. The continuum mechanics results in general tend to overestimate the natural frequencies. The two approach agree more for the cantilevered boundary condition compared with the bridged boundary condition. The results obtained in the paper may be useful for the design and analysis of vibrating SLGS based NEMS and sensor devices.

\section{Acknowledgments}

RC acknowledges the support of Royal Society through the award of Newton International Fellowship. SA gratefully acknowledges the support of The Leverhulme Trust for the award of the Philip Leverhulme Prize.

\section{References}

[1] Gan Y, Chu W and Qiao L 2003 STM investigation on interaction between superstructure and grain boundary in graphite Surf. Sci. 539 120-8

[2] Novoselov K S, Geim A K, Morozov S V, Jiang D, Zhang Y, Dubonos S V, Grigorieva I V and Firsov A A 2004 Electric field effect in atomically thin carbon films Science 306 666-9

[3] Novoselov K S et al 2005 Two-dimensional gas of massless Dirac fermions in graphene Nature 438 197-200

[4] Chowdhury R, Adhikari S, Rees P, Wilks S P and Scarpa F 2011 Graphene-based biosensor using transport properties Phys. Rev. B 83045401

[5] Huang Y, Wu J and Hwang K C 2006 Thickness of graphene and single wall carbon nanotubes Phys. Rev. B 74245413

[6] Jensen K, Kim K and Zettl A 2008 An atomic-resolution nanomechanical mass sensor Nature Nanotechnol. $3533-7$
[7] Seoanez C, Guinea F and Castro Neto A H 2007 Dissipation in graphene and nanotube resonators Phys. Rev. B 76125427

[8] Bao W, Miao F, Chen Z, Zhang H, Jang W, Dames C and Lau C N 2009 Controlled ripple texturing of suspended graphene and ultrathin graphite membranes Nature Nanotechnol. 4562

[9] Cranford S, Dipanjan S, Buehler M J and 2009 Meso-origami: folding multilayer graphene sheets App. Phys. Lett. 951231121

[10] Lee C, Wei X, Kysar J W and Hone J 2008 Measurement of the elastic properties and intrinsic strength of monolayer graphene Science $321385-8$

[11] Scarpa F, Adhikari S, Gil A J and Remillat C 2010 The bending of single layer graphene sheets: Lattice versus continuum approach Nanotechnology 21125702

[12] Schwartzbart M, Steindl A and Troger H 2008 Molecular statical calculation of graphene sheet buckling Proc. Appl. Math. Mech. 810343

[13] Duan W H and Wang C M 2009 Nonlinear bending and stretching of a circular graphene sheet undera central point load Nanotechnology 20075702

[14] Arsat R, Breedon M, Shafiei M, Spizziri P G, Gilje S, Kaner R B, Kalantar-Zadeh K and Wlodarski W 2009 Graphene-like nano-sheets for surface acoustic wave gas sensor applications Chem. Phys. Lett. 467 344-7

[15] Rangel N L and Seminario J A 2008 Graphene terahertz generators for molecular circuits and sensors J. Phys. Chem. A 112 13699-705

[16] Mohanty N and Berry V 2008 Graphene-based single-bacterium resolution biodevice and DNA transistor: interfacing graphene derivatives with nanoscale and microscale biocomponents Nano Lett. 8 4469-76

[17] Moradian R, Mohammadi Y and Ghobadi N 2008 Investigation of gas sensing properties of armchair graphene nanoribbons J. Phys. Condens. Matter 20425211

[18] Hadlington S 2007 Graphene sensor achieves ultimate sensitivity Chem. World 4 (9) 29

[19] Kitipornchai S, He X Q and Liew K M 2005 Continuum model for the vibration of multilayered graphene sheets Phys. Rev. B 72075443

[20] Hashemnia K, Farid M and Vatankhah R 2009 Vibrational analysis of carbon nanotubes and graphene sheets using molecular structural mechanics approach Comput. Mater. Sci. 47 79-85 
[21] Gupta S S and Batra R C 2010 Elastic properties and frequencies of single-layer graphene sheets J. Comput. Theor. Nanosci. 7 (10) 2151-64

[22] Hemmasizadeh A, Mahzoon M, Hadi E and Khandan R A 2008 Method for developing the equivalent continuum model of a single layer graphene sheet Thin Solids Films 516 (21) 7636-40

[23] Batra R C and Sears A 2007 Continuum models of multi-walled carbon nanotubes Int. J. Solids Struct. $447577-96$

[24] Arroyo M and Belytschko T 2003 Nonlinear mechanical response and rippling of thick multiwalled carbon nanotubes Phys. Rev. Lett. 91215505

[25] Lu Q, Arroyo M and Huang R 2009 Elastic bending modulus of monolayer graphene J. Phys. D: Appl. Phys. 42102002

[26] Odegard G M, Gates T S, Nicholson L M and Wise K E 2002 Equivalent-continuum modeling of nano-structured materials Compos. Sci. Technol. 62 1869-80

[27] Chowdhury R, Wang C Y and Adhikari S 2010 Low frequency vibration of multiwall carbon nanotubes with heterogeneous boundaries J. Phys. D: Appl. Phys. 43085405

[28] Tserpes K I and Papanikos P 2005 Finite element modelling of single-walled carbon nanotubes Composites B 36468

[29] Scarpa F, Adhikari S and Phani A S 2009 Effective elastic mechanical properties of single layer graphene sheets Nanotechnology 20065709

[30] Scarpa F, Adhikari S and Wang C Y 2009 Mechanical properties of non reconstructed defective single wall carbon nanotubes J. Phys. D: Appl. Phys. 42142002

[31] Sadeghi M and Naghdabadi R 2010 Nonlinear vibrational analysis of single-layer graphene sheets Nanotechnology 21105705

[32] Chowdhury R, Adhikari S, Wang C Y and Scarpa F 2010 A molecular mechanics approach for the vibration of single walled carbon nanotubes Comput. Mater. Sci. 48 730-35

[33] Scarpa F, Ruzzene M, Adhikari S and Chowdhury R 2010 Wave propagation and structural dynamics in graphene nanoribbons SPIE 76461A 76461A

[34] Reddy C D, Rajendran S and Liew K M 2006 Equilibrium configuration and elastic properties of finite graphene Nanotechnology 17864
[35] Rajendran S and Reddy C D 2006 Determination of elastic properties of graphene and carbon-nanotubes using brenner potential: the maximum attainable numerical precision J. Comput. Theor. Nanosci. 33 382-90

[36] Adhikari S and Chowdhury R 2010 The calibration of carbon nanotube based bio-nano sensors J. Appl. Phys. 107124322

[37] Chowdhury R, Adhikari S and Scarpa F 2011 Vibrational analysis of $\mathrm{ZnO}$ nanotubes: A molecular mechanics approach Appl. Phys. A 102 301-8

[38] Sakhaee-Pour A, Ahmadian M T and Naghdabadi R 2008 Vibrational analysis of single-layered graphene sheets Nanotechnology 19085702

[39] Bathe K J 1995 Finite Element Procedures (Englewood Cliffs, NJ: Prentice Hall)

[40] Timoshenko S 1940 Theory of Plates and Shells (New York: McGraw-Hill)

[41] DiBiasio C M, Cullinan M A and Culpepper M L 2007 Difference between bending and stretching moduli of single-walled carbon nanotubes that are modeled as an elastic tube Appl. Phys. Lett. 90203116

[42] Huang Y, Wu J and Hwang K C 2006 Thickness of graphene and single-wall carbon nanotubes Phys. Rev. B 74245413

[43] Soedel W 2004 Vibrations of Shells and Plates 3rd edn (New York: Marcel Dekker)

[44] Blevins R D 1984 Formulas for Natural Frequency and Mode Shape (Malabar, FL: Krieger)

[45] Rappe A K, Casewit C J, Colwell K S, Goddard W A and Skiff W M 1992 UFF, a full periodic-table force-field for molecular mechanics and molecular-dynamics simulations J. Am. Chem. Soc. 114 10024-35

[46] Frisch M J et al Gaussian 09 Revision A.1.

[47] McIntosh D F 2010 The determination of Wilson-Decius F matrix elements from Cartesian force constants Theor. Chem. Acc.: Theory, Comput. Modeling 125 177-84

[48] Reddy C D, Ramasubramaniam A, Shenoy V B and Zhang Y W 2009 Edge elastic properties of defect-free single-layer graphene sheets Appl. Phys. Lett. 94101904 